\title{
Magnetic Properties of Co-Fe-Si-B Microwires
}

\author{
P. Kwapuliński ${ }^{a, *}$, J. Gieraltowski ${ }^{b}$, C. Dolabdjian ${ }^{c}, Z_{\text {Z. Stokeosa }}^{a}$ And G. HaneCzok ${ }^{a}$ \\ ${ }^{a}$ University of Silesia in Katowice, Institute of Materials Science, 75 Pułku Piechoty 1A, 41-500 Chorzów, Poland \\ ${ }^{b}$ LDO/IUEM UMR CNRS 6538, UEB, Université de Bretagne Occidentale, Place Copernic, Technopôle, \\ Brest-Iroise, 29280 Plouzané, France \\ ${ }^{c}$ Normandie Université, UCBN, GREYC, CNRS, UMR 6072, F-14032 Caen, France
}

\begin{abstract}
In the paper the magneto-impedance effect in the $\mathrm{Co}_{68.15} \mathrm{Fe}_{4.35} \mathrm{Si}_{12.55} \mathrm{~B}_{15}$ microwire with diameter of about $100 \mu \mathrm{m}$ is carefully studied. The measurements in close contact and contactless geometry were carried out in the static field ranging from 0 to $6 \mathrm{kA} / \mathrm{m}$ and frequencies of the alternating field from $20 \mathrm{~Hz}$ to $2 \mathrm{MHz}$. It is shown that the examined microwire shows high anisotropy of soft magnetic properties i.e. the circumferential permeability is at least 7 times higher than the longitudinal one. Moreover, the microwire can be used as highly sensitive magneto-impedance sensor working in contactless geometry especially at fields below $200 \mathrm{~A} / \mathrm{m}$.
\end{abstract}

DOI: 10.12693/APhysPolA.131.1256

PACS/topics: $72.15 \mathrm{Gd} ; 75.30 \mathrm{Gw} ; 75.50 \mathrm{Gg} ; 75.50 \mathrm{Kj} ; 75.90+\mathrm{w}$

\section{Introduction}

$\mathrm{Fe}-\mathrm{Co}-\mathrm{Si}-\mathrm{B}$ amorphous and nanocrystalline alloys are very promising materials because of a lot of potential applications. For example these alloys with a high Co content show a relatively high Curie temperature and can be used as soft magnets at elevated temperatures even over $800 \mathrm{~K}[1]$. It is especially interesting that these alloys due to a high degree of electron-phonon anharmonicity are proposed as photo-induced nonlinear optical materials [2]. Moreover, cobalt based amorphous microwires show a giant magneto-impedance effect and therefore can be applied as magnetic sensors of very high sensitivity [37]. The goal of the present paper is to study magnetic properties of the amorphous $\mathrm{Co}_{68.15} \mathrm{Fe}_{4.35} \mathrm{Si}_{12.55} \mathrm{~B}_{15}$ microwire with diameter of about $100 \mu \mathrm{m}$. Special attention is paid to the magneto-impedance effect which corresponds to a change of electrical impedance $Z$ (measured at alternating field $H_{a c}$ ) of a ferromagnetic sample caused by application of an external static magnetic field $H_{d c}$. In typical experiments the field $H_{d c}$ acts along the wire axis and the field $H_{a c}$ is applied in two ways: (i) in the close contact method (CC) the alternating current passes through the sample which means that the circular field $H_{a c}$ is perpendicular to $H_{d c}$, and (ii) in the contactless method (CL) the alternating field $H_{a c}$ comes from an external coil i.e. $H_{a c}$ is parallel to $H_{d c}$. It is proper to add that the magneto-impedance effect is mostly studied by applying the $\mathrm{CC}$ method only while it is obvious that a simultaneous application of CC and CL methods gives a full material characteristic. Indeed, magnetization processes in both of these cases are essentially different and their comparative studies are important from a scientific as well as a practical point of view. Moreover a determination of magneto-impedance parameters (field,

*corresponding author; e-mail: piotr.kwapulinski@us.edu.pl frequency, sensitivity) allows a proper magnetic sensor design. Regardless of the method used in experiments, the magneto-impedance effect is quantitatively defined as a relative change of material impedance $Z$ due to application of the field $H_{d c}[6]$ :

$$
\Delta Z / Z=\frac{\left|Z\left(H_{d c}\right)\right|-\left|Z\left(H_{d c \max }\right)\right|}{\left|Z\left(H_{d c \max }\right)\right|} \times 100 \%,
$$

where $Z\left(H_{d c}\right)$ is the impedance determined at field $H_{d c}$ and $Z\left(H_{d c \max }\right)$ is the impedance determined at maximum of the applied field $H_{d c}$. The sensitivity of magnetoimpedance effect to changes of magnetic field $H_{d c}$, usually denoted as $\xi$, is defined as [7]:

$$
\xi=2(\Delta Z / Z)_{\max } / \Delta H_{d c},
$$

where $\Delta H_{d c}$ is the halfwidth of $\Delta Z / Z$ maximum plotted vs. $H_{d c}$. In close contact measurements the penetration depth $\delta$ is given by [6]:

$$
\delta=\frac{1}{\sqrt{\pi \mu_{\phi} \mu_{0} f \sigma}}
$$

where $\mu_{\phi}$ is the relative circumferential magnetic permeability, $\sigma$ is the electrical conductivity, $f$ is the frequency and $\mu_{0}$ is the free space permeability. It is proper to add that in our case for $f=10 \mathrm{kHz}$ the value of $\delta$ is approximately $110 \mu \mathrm{m}$. For $\delta>R(R$ is the wire radius $\approx 50 \mu \mathrm{m})$ the imaginary component $X$ of the impedance $Z$ can be expressed by [8]:

$$
X=0.175 \mu_{0} f l \mu_{\phi},
$$

where $l$ is the sample length. Equation (4) may be used for determination of the circumferential permeability which should be distinguished from the longitudinal magnetic permeability $\mu$ measured in contactless geometry i.e. when the magnetic field is parallel to the sample axis. In this case, $X=\omega L$ where $\omega$ is the angular frequency and $L$ is the coil inductance proportional to $\mu$.

\section{Results and discussion}

Measurements of magnetoimpedance effect in contact and contactless geometry were carried out by applying 
precision RLC Agilent E4980A meter working in a frequency range from $20 \mathrm{~Hz}$ to $2 \mathrm{MHz}$. Homogeneity of the $\mathrm{Co}_{68.15} \mathrm{Fe}_{4.35} \mathrm{Si}_{12.55} \mathrm{~B}_{15}$ amorphous microwires was tested using resistivity measurements and it was shown that this quantity is stable along the wire length with the precision better than 1\%. For magneto-impedance experiments sample length and diameter were about $2 \mathrm{~cm}$ and $100 \mu \mathrm{m}$, respectively.

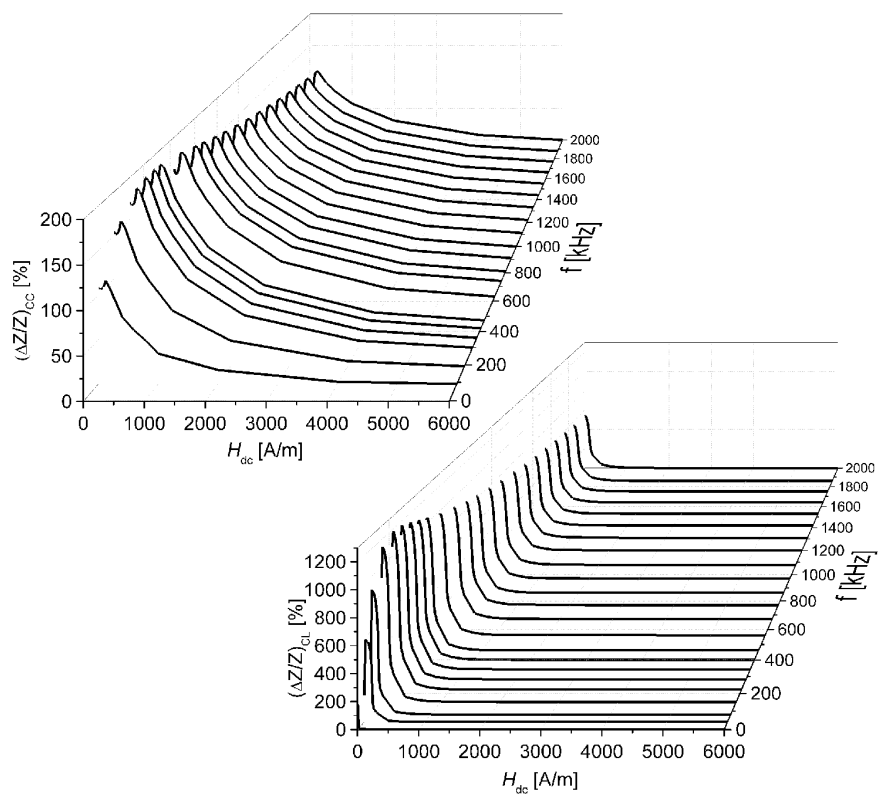

Fig. 1. Magneto-impedance effect $\Delta Z / Z$ vs. frequency $f$ and applied magnetic field $H_{d c}$; top - close contact geometry $(\Delta Z / Z)_{c c}$ with $I_{a c}=0.05 \mathrm{~mA}$ and bottom contactless geometry $(\Delta Z / Z)_{c l}$ with $H_{a c}=0.5 \mathrm{~A} / \mathrm{m}$.

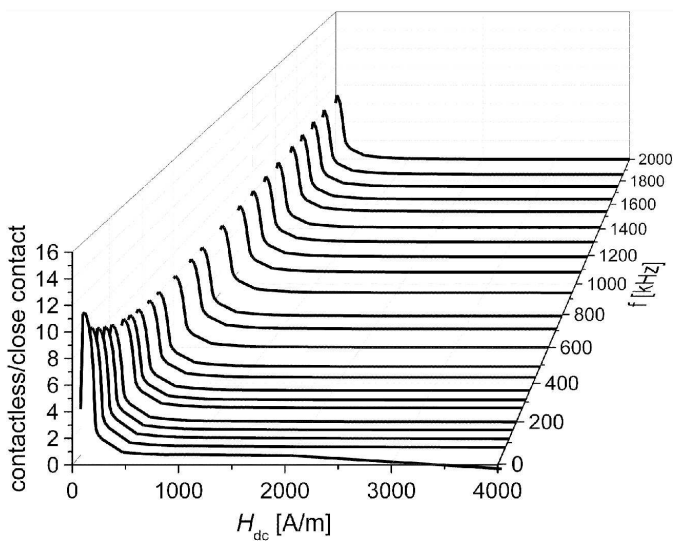

Fig. 2. Ratio $(\Delta Z / Z)_{c c}$ determined for $I_{a c}=0.05 \mathrm{~mA}$.

Figure 1 presents $\Delta Z / Z$ measured by applying the close contact $(\Delta Z / Z)_{c c}$ and contactless $(\Delta Z / Z)_{c l}$ method plotted versus frequency $f(1 \mathrm{kHz} \leq f \leq 2 \mathrm{MHz})$ of alternating magnetic field $H_{a c}$ and static magnetic field $H_{d c}$.

Figure 2 shows the ratio of contactless and close contact magneto-impedance effect $(\Delta Z)_{c l} /(\Delta Z)_{c c}$ plotted versus field $H_{d c}$ for the applied alternating current

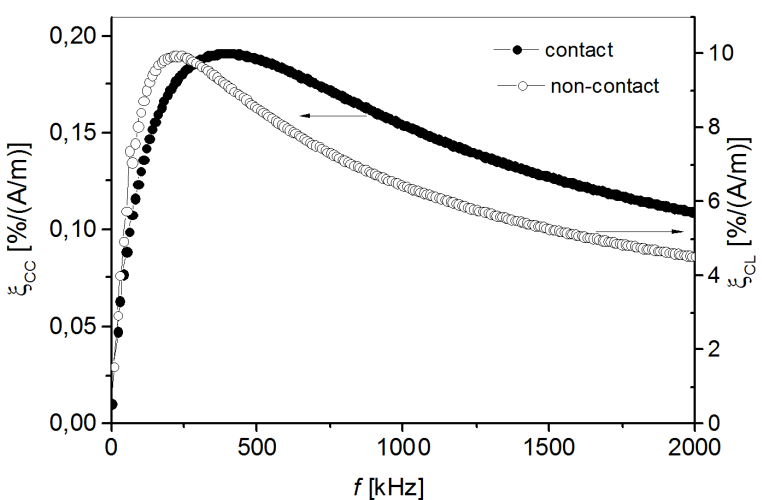

Fig. 3. Magneto-impedance sensitivity $\xi$ versus frequency for close contact and contactless measurements, $I_{a c}=0.05 \mathrm{~mA}$ and $H_{a c}=0.5 \mathrm{~A} / \mathrm{m}$.

$I_{a c}=0.05 \mathrm{~mA}$. According to the presented data for $H_{d c} \leq 200 \mathrm{~A} / \mathrm{m}$, the $(\Delta Z / Z)_{c l}$ effect is at least 7 times higher than $(\Delta Z / Z)_{c c}$.

Figure 3 shows magneto-impedance sensitivity $\xi$ plotted versus frequency for close contact and contactless measurements. Note that in both cases $\xi$ shows a maximum located at a frequency depending on the method used in measurements - i.e. at about $200 \mathrm{kHz}$ for $\xi_{c l}$ and at about $300 \mathrm{kHz}$ for $\xi_{c c}$. At this maximum, $\xi_{c l}$ is about 50 times higher than $\xi_{c c}$. These facts suggest that application of contactless method of measurements allows one to improve the sensitivity of a magneto-impedance sensor especially at relatively low magnetic fields i.e. below $200 \mathrm{~A} / \mathrm{m}$. It has to be stressed that in (CC) geometry, $H_{a c}$ inside the wire is proportional to $r(0 \leq r \leq R)$ which means that the magnetic field is from the range $0-i /(2 \pi R)$ with the mean value $i /(4 \pi R)$. In our case, the current we have used is in the range $0.01-10 \mathrm{~mA}$ which corresponds to a change of magnetic field from $0.05 \mathrm{~A} / \mathrm{m}$ to $15 \mathrm{~A} / \mathrm{m}$. This distribution of $H_{a c}$ explains a substantially lower sensitivity of a magneto-impedance effect to a change of the magnetic field in close contact measurements, as demonstrated in Fig. 3.

Figures 4 and 5 show low field circumferential permeability $\mu_{\varphi}$ (determined at $I_{a c}=0.05 \mathrm{~mA}$ ) and longitudinal magnetic permeability $\mu$ (determined at $H_{a c}=$ $0.1 \mathrm{~A} / \mathrm{m}$ ) plotted versus $H_{d c}$ for different frequencies. In both cases a maximum at fields below $200 \mathrm{~A} / \mathrm{m}$ is observed. According to Fig. 4, the low field circumferential permeability $\mu_{\varphi}$ is at least 7 times higher than the longitudinal permeability $\mu$. Such an anisotropy of soft magnetic properties means that magnetization of the examined wire via application of the circular magnetic field (CC method) is much easier than via application of a magnetic field oriented along the sample axis. This effect can be explained by taking into account that the uniaxial stress acting during the microwire production process introduces into the examined material an induced magnetic anisotropy and therefore the inter part is magnetically much harder [9]. Indeed, according to $[9,10]$ the circumferential magnetization is associated with subsur- 


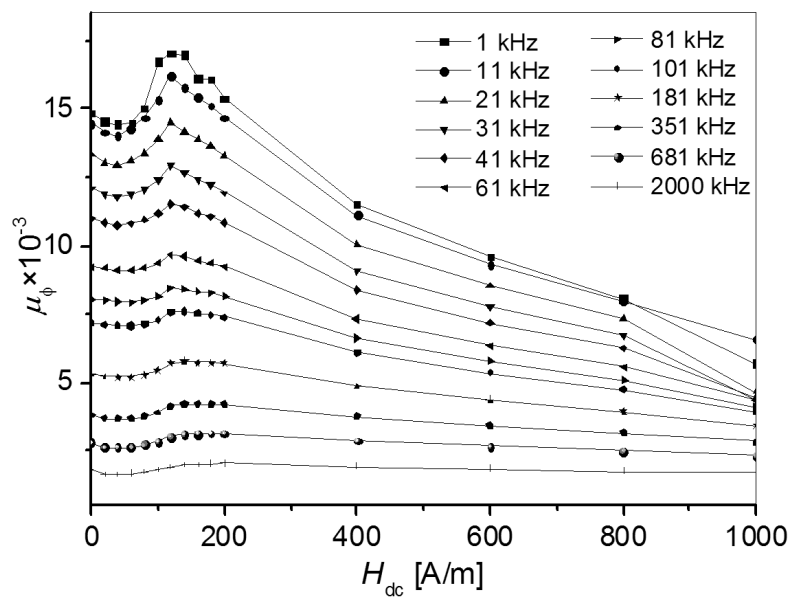

Fig. 4. Circumferential permeability $\mu_{\varphi} \quad\left(I_{a c}=\right.$ $0.05 \mathrm{~mA}$ ) versus $H_{d c}$ for different frequencies.

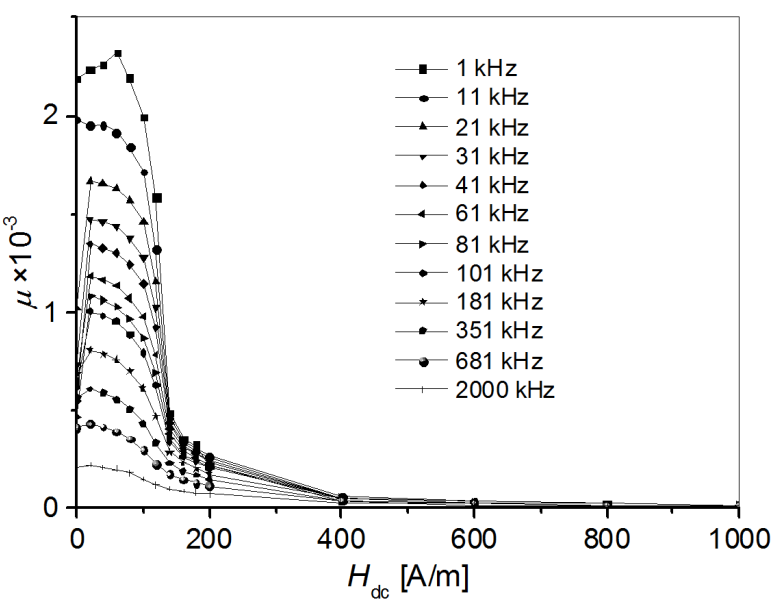

Fig. 5. Magnetic permeability $\mu\left(H_{a c}=0.5 \mathrm{~A} / \mathrm{m}\right)$ plotted versus $H_{d c}$ for different frequencies.

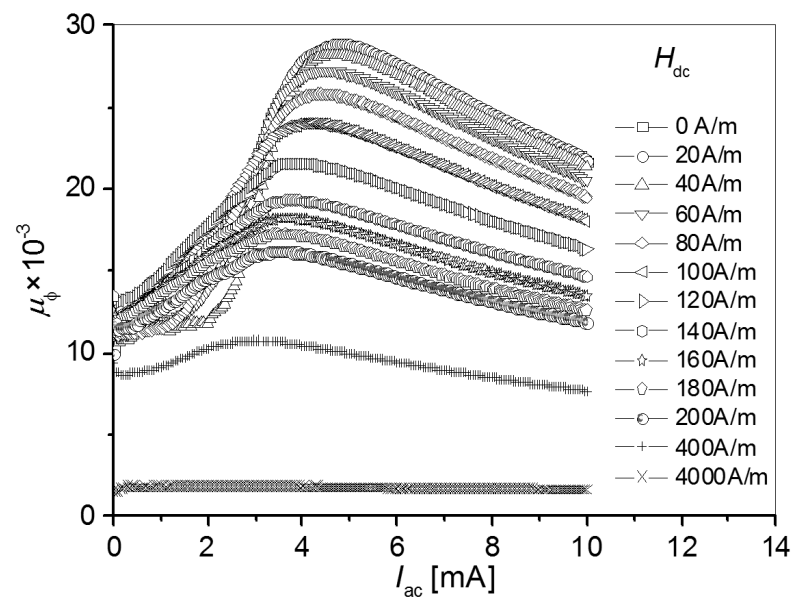

Fig. 6. Circumferential permeability $\mu_{\varphi}$ versus $I_{a c}$ determined at $f=10 \mathrm{kHz}$ for different direct magnetic fields $H_{d c}$.

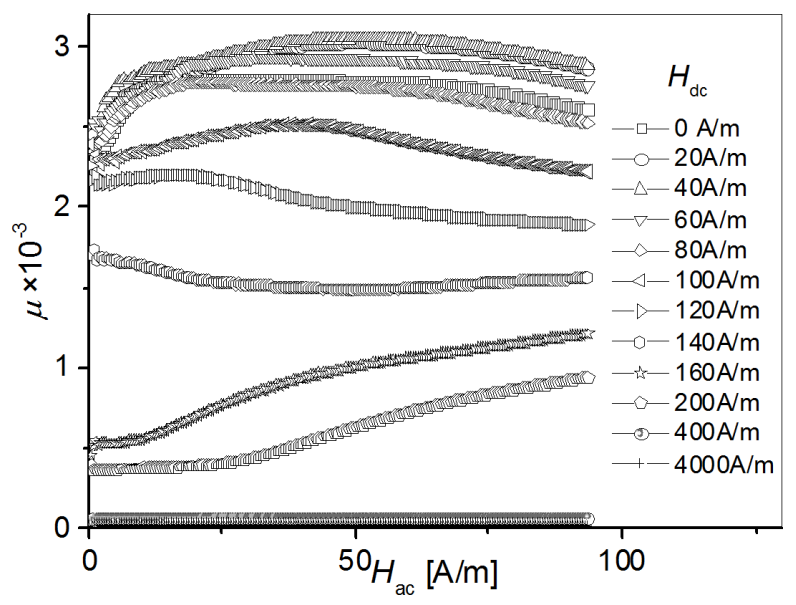

Fig. 7. Permeability $\mu$ versus $H_{a c}$ determined at frequency $f=10 \mathrm{kHz}$ for different direct magnetic fields $H_{d c}$.

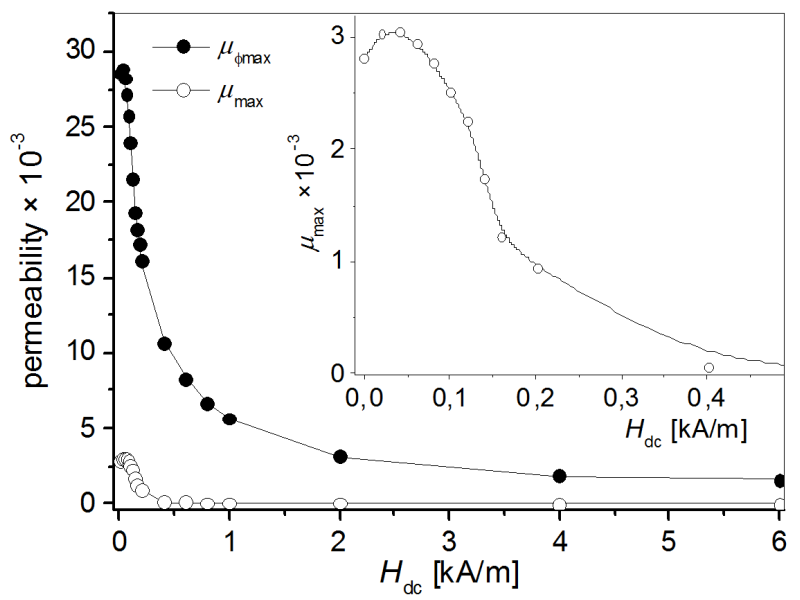

Fig. 8. Maximum of permeability from Figs. 6 and 7 plotted versus $H_{d c}$; in the inset $\mu_{\max }$ in weak fields is shown.

face cylindrical magnetic domains and in contrast to this magnetization along the wire axis should be associated with magnetic domains lying inside, along the axis of the wire. Finally, it can be concluded that due to a specific domain structure the outer part of the microwire is magnetically much more softer.

The discussed magnetic anisotropy is also presented in Figs. 6 and 7 where $\mu_{\varphi}$ and $\mu$ are plotted versus amplitude of the alternate magnetic field $H_{a c}$ for different $H_{d c}$ and one selected frequency $1 \mathrm{kHz}$. Again, it can be concluded that in all studied cases $\mu_{\varphi}$ is much higher than $\mu$ which reflects different geometry used in experiments and that follows different soft magnetic properties of the inner and outer part of the examined wire.

The value of magnetic permeability at maximum of Figs. 6 and 7 is plotted versus magnetic field $H_{d} c$ in Fig. 8. One can see that the sensitivity of the studied material to changes of the static magnetic fields is much higher for contactless measurements although the corresponding permeability is much lower. 


\section{Conclusions}

The experimental results presented in this paper referring to the amorphous $\mathrm{Co}_{68.15} \mathrm{Fe}_{4.35} \mathrm{Si}_{12.55} \mathrm{~B}_{15}$ microwire can be summarized as follows: (i) soft magnetic properties show very high anisotropy i.e. the low field circumferential permeability is at least 7 times higher than the low field longitudinal permeability (see Figs. 4 and 5), (ii) magneto-impedance effect measured by applying the contactless method is about 7 times higher than measured by applying the close contact method (see Figs. 1 and 2),

(iii) sensitivity of magneto-impedance effect plotted versus frequency shows a maximum located at $200 \mathrm{kHz}$ and $300 \mathrm{kHz}$ for contactless and close contact measurements, respectively, (iv) the contactless sensitivity of the magneto-impedance effect is about 50 times higher than the close contact one which means that the examined wire can be used as a magnetic sensor working in contactless geometry especially at low magnetic fields i.e. below $200 \mathrm{~A} / \mathrm{m}$ (see Figs. 3 and 5-8).

\section{References}

[1] T. Kulik, J. Ferenc, A. Kolano-Burian, X.B. Liang, M. Kowalczyk, J. Alloys Comp. 434-435, 623 (2007).
[2] I.V. Kityk, E. Jakubczyk, Appl. Opt. 38, 5162 (1999).

[3] J.J. Beato-López, C.A. de la Cruz Blas, A. Mitra, C. Gómez-Polo, Sensor Actuat. A Phys. 242, 73 (2016).

[4] R. Valenzuela, A. Fessant, J. Gieraltowski, C. Tannous, Sensor Actuat. A Phys. 142, 533 (2008).

[5] B. Dufay, S. Saez, C. Dolabdjian, A. Yelon, D. Menard, J. Magn. Magn. Mater. 324, 2091 (2012).

[6] A. Zhukov, M. Ipatov, V. Zhukova, in: 0.1016/bs.hmm.2015.09.001Handbook of Magnetic Materials, Vol. 24, Ch. 2, Elsevier, 2015, p. 139.

[7] A. Amirabadizadeh, Z. Lotfollahi, A. Zelati, J. Magn. Magn. Mater. 415, 102 (2016).

[8] M. Knobel, M.L. Sanchez, C. Gomez-Polo, P. Marın, M. Vazquez, A. Hernando, J. Appl. Phys. 79, 1646 (1996).

[9] L.V. Panina, K. Mohri, T. Uchizama, M. Noda, IEEE Trans. Magn. 31, 1249 (1995).

[10] V. Zhukova, N.A. Usov, A. Zhukov, J. Gonzalez, Phys. Rev. B 65, 134407 (2002). 